\title{
Study on the Features of Coronary Artery Atheromatous Plaque for Patients with Impaired Glucose Tolerance when Applying Intravascular Ultrasound
}

\author{
Jinming Cen', Qingyuan Xiong ${ }^{2}$, Xilin Yang ${ }^{1 *}$ and Zhaoyan $\mathbf{X u}^{\mathbf{1}}$ \\ ${ }^{1}$ The Cardiology Department of the 1st People Hospital of Foshan, China \\ ${ }^{2}$ Department of Clinical Laboratory, The Chancheng District Central Hospital of Foshan, China
}

\begin{abstract}
Objective: By applying Intravascular Ultrasound (IVUS) to patients with mild-to-moderate coronary stenosis defined by Coronary Angiography (CAG), it is analyzed on the features of coronary artery atheromatous plaque for patients with impaired glucose tolerance and discussed about the clinical significance as well as the relationship between $\mathrm{HbA} 1 \mathrm{c}$ levels and coronary artery lesion.
\end{abstract}

Methods: HbA1c testing was applied to 85 patients (a total of 96 lesions) with 46 cases in Group of Impaired Glucose Tolerance (IGT Group) and 39 cases in Group of Normal Blood Glucose (NBG Group). IVUS was applied to qualitatively and quantitatively analyse the lesion vessel of both groups. Measurement was done both to target lesion for data of External Elastic Membrane Area (EEMA), Minimal Lumen Area (MLA), Plaque Area (PA), Plaque Burden $(\mathrm{PB})$, and to the reference segments for data of Reference External Elastic Membrane Area (REEMA), Minimal Lumen Area (RMLA), Plaque Area (RPA), Plaque Burden (RPB).

Results: The level of HbA1c in IGT Group was significantly higher than that in NBG Group $(P<0.05)$. For IGT Group, there were more soft plaque, eccentric plaque, positive remodeling and less calcification while for NBG Group, there were more performance for the hard plaque, calcification, no reconstruction and negative remodeling $(P<0.05)$. For IGT Group, MLA was less than NBG Group while EEMA, PA and PB were obviously higher than NBG Group $(P<0.05)$. In the meantime, RMAL was clearly less than NBG Group while RPA and RPB were higher than NBG Group $(P<0.05)$. HbA1c levels was positively correlated with PA, PB, but negatively correlated with MLA.

Conclusion: IVUS shows a higher value to the evaluation of mild-to-moderate coronary lesions. The coronary artery lesions of IGT Group were more serious and widespread than that of NBG Group, and the level of HbA1c might provide some value for the judgment of the severity of coronary artery lesion.

Keywords: Atherosclerosis; Intravascular ultrasound; Coronary angiography; Impaired glucose tolerance

\section{Introduction}

Impaired glucose tolerance is a state resulting from changes of blood glucose on the early steady state, which greatly increases the risk of microvascular and macrovascular complications. Patients will suffer significantly increasing risk of serious adverse cardiovascular events when there is a co-existence of impaired glucose tolerance and coronary atherosclerosis [1].

In recent years, researches showed that the severity of atherosclerotic lesion only shared weak correlation with its clinical events, the majority ( $60 \%-70 \%)$ of which was causing by the rupture of atherosclerotic plaque and the secondary thrombus. It was found, with traditional coronary angiography, that most of the clinical events were mild-to-moderate stenosis [2-4]. In this research, CAG was used to test patients having both coronary artery disease and impaired glucose tolerance. For those cases that showed mild-to-moderate stenosis in CAG, IVUS was applied to recognize pathological changes of coronary plaque, and then the clinical significance was discussed.

\section{Subjects and Methods}

Subjects from July 2009 to December 2010, 85 cases were collected including 46 cases of impaired glucose tolerance (IGT Group) and 39 cases of Normal Blood Glucose (NBG Group), a total of 96 lesions. Both groups were taken CAG and confirmed that the stenosis rate of at least one epicardial coronary and its main branch vessel diameter was $50 \% \sim 70 \%$. Both groups were not taking hypoglycemic drugs.

\section{Analysis indicators of coronary angiography images}

Confirm the normal reference segments and the lesion vessel segments. "Normal" reference segment was defined to vessel without pathological changes within $5 \mathrm{~mm} \sim 10 \mathrm{~mm}$ apart from the proximal or distal of the target lesion vessel, while the lesion segment refers to the most stenosis according to angiography images from different projected position, which was also called Quantitative Coronary Angiography (QCA) [5-8].

\section{Analysis indicators of IVUS images}

Quantitative indicators: All testing data involved was collected from the most stenosis segment showing by IVUS, including EEMA, MLA, PA, PB and vascular calcification, etc. The other indicators referred to the reference segment, including REEMA, RMLA, RPA, RPB. Qualitative indicators: Depending on ultrasonic echo, plaque can

*Corresponding author: Xilin Yang, The Cardiology Department of the 1st People Hospital of Foshan, Guangdong 528000, China, Tel: 0757-8316 3155; E-mail: 289124834@qq.com

Received February 23, 2016; Accepted March 08, 2016; Published March 14 2016

Citation: Cen J, Xiong Q, Yang X, Xu Z (2016) Study on the Features of Coronary Artery Atheromatous Plaque for Patients with Impaired Glucose Tolerance when Applying Intravascular Ultrasound. Cardiovasc Pharm Open Access 5: 177. doi:10.4176/2329-6607.1000177

Copyright: (c) 2016 Cen J, et al. This is an open-access article distributed under the terms of the Creative Commons Attribution License, which permits unrestricted use, distribution, and reproduction in any medium, provided the original author and source are credited. 
be divided into four types: soft plaque, fibrous plaque, calcific plaque and mixed plaque. Eccentric plaque refers to plaque that builds up itself on the wall of the arteries or plaque whose thickest part is more than twice of its thinnest one [9-11].

\section{Statistical methods}

SPSS statistical software was employed. Chi-square test was used to make the comparison among enumeration data while measurement data was showed by mean. T-test was applied to make contract. Pearson correlative analysis was adopted to show the correlation between those two factors, and $\mathrm{P}<0.05$ presented the significant difference.

\section{Results}

From the clinical records of both groups, it was found that, in terms of the level of glycated hemoglobin (HbAlc), IGT Group was significantly higher than NBG Group $(\mathrm{P}<0.05)$ (Table 1).

Following up 96 pathologic changes, for IGT Group, MLA was less than NBG Group while EEMA, PA and PB were obviously higher than NBG Group $(\mathrm{P}<0.05)$. However, RMAL was clearly less than NBG Group while RPA and RPB were higher than NBG Group $(\mathrm{P}<0.05)$ (Table 2).

In IGT Group, there were 52 plaque including 36 eccentric plaques being followed while in NBG Group, 44 plaques with 20 eccentric plaques, leading to statistical significance for their differences $(P=0.019)$. For IGT Group, there were more soft plaque, eccentric plaque and positive remodeling in coronary artery but less calcification while in NBG Group, it was presented as fibrous plaque, calcification, no remodeling and negative remodeling $(\mathrm{P}<0.05)$ (Table 3$)$.

Linear correlation analysis was done between levels of $\mathrm{HbAlc}$ and MLA, PA and PB $(\mathrm{P}<0.05)$ (Table 4).

Figure 1 was IVUS image with mild-to-moderate lesion displayed by $\mathrm{CAG}$.

\section{Discussion}

Compared with CAG, IVUS can provide the details of coronary plaque and vascular walls, such as the volume of the plaque, ingredient, calcification, stability, rupture, the volume as well as shape of vascular walls. There were 96 pathological changes being followed in this research and the rates of stenosis for average area were CAG: $53.68 \% \pm 2.58 \%$ and IVUS: $59.66 \% \pm 4.66 \%(\mathrm{P}<0.05)$, presenting statistical significance for differences between groups, indicating that coronary pathological changes may be underestimated in CAG testing. The following reasons may be considered: (1) there may be atherosclerosis in the reference vascular for the reason that either near-end or far-end of the reference vascular was perfectly normal, shown from CAG images but from IVUS images, it was found that there was different levels of intimal hyperplasia or even plaque in the reference vascular. (2) To protect lumen, coronary atherosclerotic vascular may have compensatory expansion, which was vascular positive remodeling. (3) CAG can only provide two-dimensional diagram of lumen structure while IVUS can exam the lumen crosssection by 360 degree. (4) Projection angle and overlapping vessels can make a difference to the outcome of CAG; (5) there may be some subjective factors of the observers. As IVUS can provide more accurate information, diagnosis can be made with the help of intravascular ultrasound when there are some doubts on stenosis diagnosed by CAG and needs to make further confirmation about whether necessary to take revascularization, or the time when angiographic results are inconsistent with clinical manifestations.

Ko and several other researchers, following 109 patients with coronary heart disease, using IVUS, found that the main feature of plaque in ACB patients is heavy burden and positive remodeling. In this research, similar conclusion was drawn in IGT Group: by following pathological changes, it was found that the Minimal Lumen Area (MLA) of IGT Group was remarkably lower than that of NBG Group while the External Elastic Membrane Area (EEMA) was notably higher than NBG Group. Thus it can be seen that both burden and area of plaque in IGT Group were obviously larger than those in NBG Group, indicating that compared with NBG patients, coronal lesion among IGT patients apparently aggravated. There were no differences presenting in Reference External Elastic Membrane Area (REEMA) between IGT Group and NBG Group, but the lumen of reference segments in IGT Group was much smaller than that of NBG Group, showing that, for IGT Group, the plaque area and plaque burden of reference segments were significantly greater than NBG Group. Similarly, there were lesions in reference segments. Compared with NBG Group, for those patients with complicated Impaired Glucose Tolerance (IGT), the lesion was extensive and all-pervading, similar to the coronary of diabetes patients.

The stability of plaque shares a direct relationship to the occurrence of acute coronary syndrome as plaque rupture accompanied with thrombosis may lead to serious myocardial ischemia, which was the main cause for acute myocardial infarction. Acute myocardial infarction may occur when coronary artery stenosis is not so serious or may, based on this lesion, result in coronary spasm, causing angina pectoris. Pathological research found that the histology feature of vulnerable plaque were large lipid core, eccentric plaque with thin-cap fibrous, large plaque area and it does not necessarily account for a large

\begin{tabular}{|c|c|c|c|}
\hline Groups & IGT Group & NBG Group & P Value \\
\hline Male / Female (case) & $38 / 8$ & $30 / 9$ & 0.514 \\
\hline Age (year) & $61.09 \pm 2.56$ & $61.44 \pm 2.50$ & 0.684 \\
\hline Smoking (case) & 28 & 25 & 0.759 \\
\hline $\begin{array}{c}\text { History of high blood } \\
\text { pressure (case) }\end{array}$ & 35 & 28 & 0.653 \\
\hline TC (mmol/L) & $4.75 \pm 1.16$ & $4.68 \pm 0.89$ & 0.719 \\
\hline TG (mmol/L) & $2.29 \pm 1.45$ & $2.18 \pm 1.98$ & 0.751 \\
\hline LDL-C (mmol/L) & $2.79 \pm 0.65$ & $2.60 \pm 0.55$ & 0.163 \\
\hline HDL-C (mmol/L) & $1.44 \pm 0.69$ & $1.63 \pm 0.63$ & 0.175 \\
\hline EF (\%) & $56 \pm 4.7$ & $55 \pm 4.18$ & 0.220 \\
\hline LAD (case) & $20(38.5 \%)$ & $19(43.2 \%)$ & 0.639 \\
\hline RCA (case) & $17(32.7 \%)$ & $14(31.8 \%)$ & 0.927 \\
\hline LCX (case) & $15(28.8 \%)$ & $11(25.0 \%)$ & 0.673 \\
\hline QCA (\%) & $53.85 \pm 1.74$ & $53.38 \pm 1.23$ & 0.128 \\
\hline HbA1c (\%) & $5.96 \pm 0.32$ & $5.4 \pm 0.26$ & 0.000 \\
\hline
\end{tabular}

Table 1: Clinical data of two groups.

\begin{tabular}{|c|c|c|c|}
\hline Groups & IGT Group & NBG Group & P Value \\
\hline EEMA $\left(\mathrm{mm}^{2}\right)$ & $14.26 \pm 0.95$ & $13.86 \pm 0.56$ & 0.016 \\
\hline MLA $\left(\mathrm{mm}^{2}\right)$ & $5.49 \pm 0.66$ & $5.86 \pm 0.46$ & 0.002 \\
\hline PA $\left(\mathrm{mm}^{2}\right)$ & $8.76 \pm 1.08$ & $8.0 \pm 0.67$ & 0.000 \\
\hline PB $(\%)$ & $61.15 \pm 4.93$ & $57.59 \pm 3.48$ & 0.000 \\
\hline REEMA $\left(\mathrm{mm}^{2}\right)$ & $13.54 \pm 1.08$ & $13.74 \pm 1.14$ & 0.351 \\
\hline RMLA $\left(\mathrm{mm}^{2}\right)$ & $9.20 \pm 0.82$ & $10.08 \pm 0.9$ & 0.000 \\
\hline RPA $\left(\mathrm{mm}^{2}\right)$ & $4.33 \pm 1.17$ & $3.66 \pm 1.31$ & 0.008 \\
\hline RPB $(\%)$ & $31.90 \pm 7.22$ & $26.11 \pm 8.19$ & 0.001 \\
\hline RI & $1.06 \pm 0.78$ & $1.01 \pm 0.78$ & 0.008 \\
\hline
\end{tabular}

Note: $\mathrm{RI}>1.05$ presents positive remodeling; $\mathrm{RI}<0.95$ presents negative remodeling, $0.95<\mathrm{RI}<1.05$ presents non-remodeling.

Table 2: Quantitative analysis on features of coronary plaque between two group. 
Citation: Cen J, Xiong Q, Yang X, Xu Z (2016) Study on the Features of Coronary Artery Atheromatous Plaque for Patients with Impaired Glucose Tolerance when Applying Intravascular Ultrasound. Cardiovasc Pharm Open Access 5: 177. doi:10.4176/2329-6607.1000177

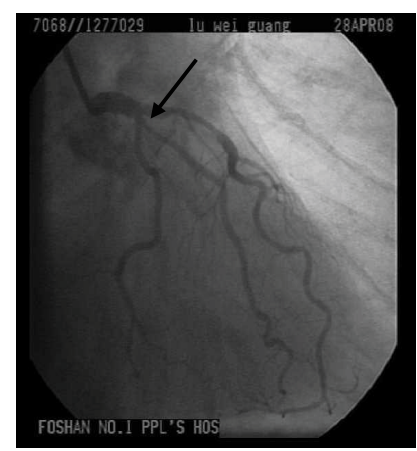

A

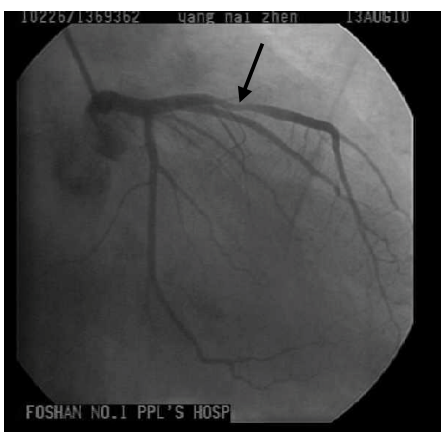

A1

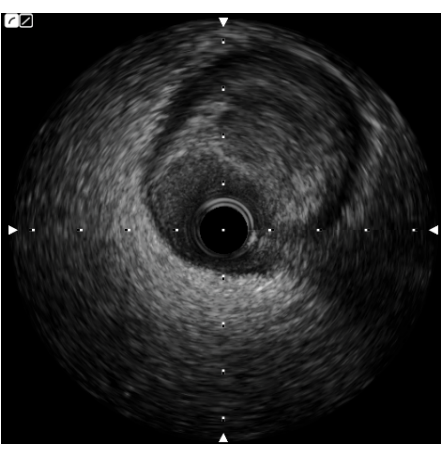

B

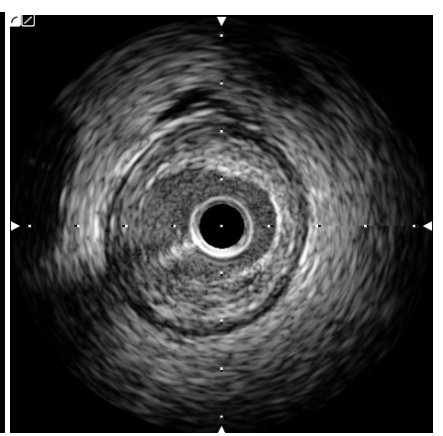

B1

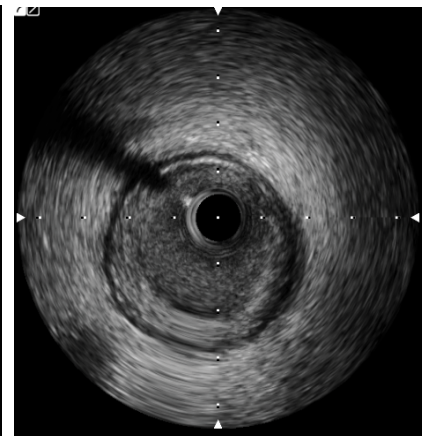

$\mathrm{C}$

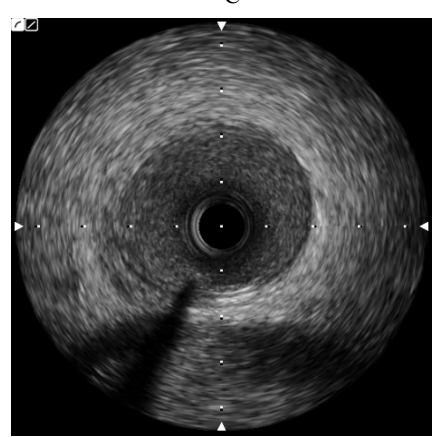

$\mathrm{C} 1$

Note: A: Near-end of LAD was eccentric and $60 \%$ stenosis. B: With IVUS, the Near-end of LAD was eccentric and soft plaque (10-3 spots); lumen area was 4.48 $\mathrm{mm}^{2}$, the rate of area stenosis was $68.14 \%$, needing intervention. C: the reference vessel of IGT Group. NBG Images: A1: Near-end of LAD was $50 \%$ stenosis. B1: IVUS shown centrality, fibrous plaque, minimal lumen area was $6.04 \mathrm{~mm}^{2}$, the rate of area stenosis was $52.31 \%$. C1: the reference vessel of NBG Group.

Figure 1: IGT Image.

\begin{tabular}{|c|c|c|c|c|c|c|c|c|c|c|c|c|}
\hline \multirow{2}{*}{ Groups } & \multirow{2}{*}{ Cases } & \multicolumn{4}{|c|}{ Plaque } & \multicolumn{4}{|c|}{$\begin{array}{c}\text { Level of } \\
\text { Calcification }\end{array}$} & \multicolumn{3}{|c|}{ Remodeling } \\
\hline & & Soft plaque & $\begin{array}{l}\text { Fibrous } \\
\text { plaque }\end{array}$ & $\begin{array}{l}\text { Calcific } \\
\text { plaque }\end{array}$ & $\begin{array}{l}\text { Mixed } \\
\text { plaque }\end{array}$ & 0 & I & II & III & $\begin{array}{l}\text { Negative } \\
\text { remodeling }\end{array}$ & $\begin{array}{l}\text { Positive } \\
\text { remodeling }\end{array}$ & Non-remodeling \\
\hline IGT Group & 52 & 28 & 14 & 5 & 5 & 32 & 12 & 7 & 1 & 12 & 27 & 13 \\
\hline NBG Group & 44 & 13 & 14 & 14 & 3 & 14 & 19 & 9 & 2 & 18 & 11 & 15 \\
\hline
\end{tabular}

Note: The visual composition of soft plaque, fibrous plaque or calcific plaque is more than $80 \%$, then the plaque was be defined as soft plaque, fibrous plaque or calcific plaque; otherwise, as mixed plaque. Level 0: Non-calcification; Level I: Calcification less than $90^{\circ}$; Level II: Calcification between $91^{\circ} \sim 180^{\circ}$; Level III: $181^{\circ} \sim 270^{\circ}$

Table 3: Qualitative comparison of coronary plaque between two groups.

\begin{tabular}{|c|c|c|}
\hline \multirow{2}{*}{ Groups } & \multicolumn{2}{|c|}{ HbAlc } \\
\cline { 2 - 3 } & r Value & P Value \\
\hline MLA & -0.395 & $<0.01$ \\
\hline PA & 0.675 & $<0.01$ \\
\hline PB & 0.615 & $<0.01$ \\
\hline
\end{tabular}

Table 4: Correlation analysis on $\mathrm{HbA1c}$ levels and MLA, PA, PB.

proportion in the lumen. Eccentric plaque and positive remodeling can offer an implication that the plaque is vulnerable for the fact that there were more lipid and macrophage in the pathological changes of positive remodeling and that to be rich in lipid and infiltrated by a large number of macrophage is the mark of plaque vulnerability. Kato [4] and others followed 110 cases of ACS patients to observe the changes of coronary artery, applying IVUS. In terms of rupture of culprit plaque, it was divided into two groups, 60 cases in Rupture Group. The remodeling index of Rupture Group was markedly higher than that of Non-rupture Group $(\mathrm{P}<0.05)$, prompting that plaque rupture is more positive remodeling. In this research, for IGT Group, there were more eccentric plaque, more lipid plaque, more positive remodeling but less calcification, indicating that for IGT patients with mild-moderate stenosis, there were many vulnerable plaque which was easy to rupture and result in acute coronary syndrome.

ARIC [5] research found that the level of HbAlc was related to the occurrence of cardiac events when $\mathrm{HbAlc}$ was greater than or equal to $6.0 \%$. Berry etc. found in their research that the increase of $\mathrm{HbAlc}$ was related to the occurrence and severity of coronary atherosclerosis, and it was beneficial to make strict control on the level of HbAlc for those coronary artery disease patients with IGT or with Diabetes Mellitus (DT). This research shows that for those who got poor control of HbAlc, the degree of their coronary artery lesion apparently aggravated, compared with those making good control. The level of HbA1c shown an increasing trend with the severity of coronary artery lesion, presenting that $\mathrm{HbAlc}$ can serve as a warning predictor for the progresses of coronary heart disease but it needs to be drawn with the assist of other examinations, such as clinical findings, electrocardiogram, coronary arteriography. What kind of mechanism that HbAlc plays in the development of coronary artery lesions still remains to be definite, and the possible related factors are as follows: 1. HbAlc can 
Citation: Cen J, Xiong Q, Yang X, Xu Z (2016) Study on the Features of Coronary Artery Atheromatous Plaque for Patients with Impaired Glucose Tolerance when Applying Intravascular Ultrasound. Cardiovasc Pharm Open Access 5: 177. doi:10.4176/2329-6607.1000177

make red blood cell with more viscosity, less liquidity and much less deformability. 2. Moreover, HbA1c can slow down the dissociation of oxyhemoglobin, increase the affinity of erythrocyte for oxygen, note worthily decrease the number of 2,3-diphosphoglyceric acid(2,3-DPG) and result in hypoxia for both tissue and cell. 3. With the increase of $\mathrm{HbA1c}$, for one thing, it rise the release of endothelin, but reduce that of Nitric Oxide (NO) and prostacyclin, damaging vasomotor capacity; for another, the direct toxic effect of Glucose decreased the replication of endothelial cell (CEC) and declined its capacity to repair cells and finally gave rise to the damage of endothelial cell, promoting the formation of atherosclerosis.

Above all, IVUS shows a higher value to the evaluation of borderline lesions as using IVUS, it was found that the coronary artery lesions of IGT Group were more serious and widespread than that of NBG Group, and it could come to the conclusion that the level of $\mathrm{HbAlc}$ might provide some value for the judgment of the severity of coronary artery lesion. Therefore, for those IGT patients with an image of mildto-moderate stenosis in CAG, especially for those having a poor control of $\mathrm{HbAlc}$, it is very important to have IVUS test to provide reference for the choice of clinical treatment strategies, declining the occurrence of adverse cardiovascular events.

\section{References}

1. Decode Study Group, European Diabetes Epidemiology Group (2001) Glucose tolerance and cardiovascular mortality: comparison of fasting and 2-hour diagnose criteria. Arch Intern Med 16: 397-405.

2. Virmani R, Kologdie FD, Burke AP, Farb A, Schwartz SM (2000) Lessons from sudden coronary death: a comprehensive morphological classification scheme for atherosclerotic lesions. Arterioscler Thromb Vasc Biol 20: 1262-1275.
3. Virmani R, Burke AP, Farb A, Kolodgie FD (2006) Pathology of the vulnerable plaque. J Am Coll Cardiol 47: C13-18.

4. Okada K, Gohbara KHM, Kataoka S, Takano K (2015) Association between blood glucose variability and coronary plaque instability in patients with acute coronary syndromes. Cardiovascular Diabetology 14: 111.

5. Kato M, Dote K, Naganuma T, Sasaki S, Ueda K, et al. (2010) Clinical predictors of culprit plaque rupture assessed on intravascular ultrasound in acute coronary syndrome. Cire J 74: 1936-1942.

6. Matsushita K, Blecker S, Pazin-Filho A, Bertoni A, Chang PP, et al. (2010) The association of hemoglobin A1c with incident heart failure among people without diabetes: the atherosclerosis risk in communities study Diabetes 59: 2020-2026.

7. Kurihara O, Takano M, Seino Y, Shimizu W, Mizuno K (2015) Coronary atherosclerosis is already on-going in pre-diabetic status: Insight from intravascular imaging modalities. World Journal of Diabetes 6: 184-191.

8. Daida H, Takayama T, Hiro T, Yamagishi M, Hirayama A, et al. (2012) High $\mathrm{HbA} 1 \mathrm{c}$ levels correlate with reduced plaque regression during statin treatment in patients with stable coronary artery disease: Results of the coronary atherosclerosis study measuring effects of rosuvastatin using intravascular ultrasound in Japanese subjects(COSMOS). Cardiovascular Diabetology 11 : 87.

9. Otten R, Kline-Rogers E, Meier DJ, Dumasia R, Fang J, et al. (2005) Impact of pre-diabetic state on clinical outcomes in patients with acute coronary syndrome. Heart 91: 1466-1468.

10. Kuhl J, Jorneskog G, Wemminger M, Bengtsson M, Lundman P, et al. (2015) Long-term clinical outcome in patients with acute coronary syndrome and dysglycaemia, Cardiovascular Diabetology 14: 120.

11. Lipton JA, Can A, Akoudad S, Simoons ML (2011) The role of insulin therapy and glucose normalization in patients with acute coronary syndrome. Neth Heart J 19: 79-84. 